\title{
Application values of 3.0T magnetic resonance diffusion weighted imaging for distinguishing liver malignant tumors and benign lesions
}

\author{
RUIBIN LI, GUANGYAO WU and RUI WANG \\ Department of Diagnostic Radiology, Zhongnan Hospital of Wuhan University, Wuhan, Hubei 430071, P.R. China
}

Received August 31, 2016; Accepted July 3, 2017

DOI: $10.3892 / \mathrm{ol} .2017 .7565$

\begin{abstract}
The aim of the present study was to investigate the significance and values of 3.0T diffusion weighted imaging (DWI) to differentially diagnose benign and malignant space-occupying liver lesions. A total of 91 patients with liver space-occupying lesions (145 lesions) were admitted into Zhongnan Hospital of Wuhan University (Wuhan, China) from November 2015 to May 2016. Routine scanning, DWI and high-resolution T2-weighted imaging using spin-echo echo-planar imaging were performed on all patients, to compare the apparent diffusion coefficient (ADC) values of three regions of interest in lesions with normal liver tissue. The ADC values of malignant liver lesions compared with benign liver cysts demonstrated a statistically significant difference in low $b$-value $(\mathrm{P}<0.05)$ and there was also a significant difference between malignant lesion and hepatic cyst, hepatic hemangioma or hepatic abscess in middle b-value $(\mathrm{P}<0.05)$. The measured ADC value may be more conducive to identify the nature of the liver space-occupying lesions; as the ADC values of malignant liver lesion, liver cyst, and liver abscesses demonstrated a statistical significance in high b-value $(\mathrm{P}<0.05)$. The mean ADC values between malignant liver tumors compared with benign lesions indicated a statistically significant difference. In the present study, liver space-occupying lesions demonstrated different DWI features and ADC ranges, and 3.0T DWI may be a potential means to accurately determine the nature of lesions, identifying benign and malignant space-occupying lesions.
\end{abstract}

\section{Introduction}

Over 100 different types of liver disease have been identified in total, including malignant liver cancer, which arises from

Correspondence to: Dr Guangyao Wu, Department of Diagnostic Radiology, Zhongnan Hospital of Wuhan University, 169 Donghu Road, Wuhan, Hubei 430071, P.R. China

E-mail: guangyaowudoc@163.com

Key words: liver space-occupying lesions, magnetic resonance imaging, diffusion weighted imaging, apparent diffusion coefficient value tumors that originate in the liver, or originate in another organ and migrate to the liver. The pathological features of malignant liver cancer are rapid progression and high mortality rates, which has been the third leading cause of cancer-associated mortality worldwide (1); therefore, it is crucial to identify benign and malignant liver lesions. With the invention and application of high-field superconducting magnetic resonance imaging (MRI), in particular the clinical application of 3.0T MRI, diffusion weighted imaging (DWI) has significance in differentiating benign and malignant liver lesions (2). DWI has been observed in a variety of well-vascularized tissues, including the brain, liver, pancreas, kidney, muscle, and placenta (3-5). The basic principle of DWI is to add the symmetric diffusion-sensitive gradient pulse (b) into conventional spin echo (SE) T2-weighted scanning sequence. Based on the principles of osmotic water diffusion, water molecules are activated and migrate towards the direction of the applied gradient field (6). The greater the b-value, the more severe the phase dispersion of water molecules, and the more apparent the signal reduction. If lesions are present in organs, the dispersion degree of water molecules in normal tissue and lesions will vary, therefore the degree of signal reduction will be different, thus facilitating the detection of lesions (7). The b-value scheme also affects the DWI parameters and the separation between their values in cancer and normal tissue when the number of $b$-values is small. In the diagnosis of liver lesions, DWI imaging may be used as an adjunct technique to contrast-enhanced MRI, which provides high sensitivity. The applications of DWI technology based on this theory are currently more widely reported in liver disease research (8-11), but its value in diagnosing focal liver lesions varied among previous studies (12-16). The purpose of the present study was to employ DW MRI at 3.0T and to apply different b-values to scan liver lesions, with the aim of observing DWI features and apparent diffusion coefficient (ADC) changes of lesions with different characteristics; investigating the differential diagnostic value of DWI towards benign and malignant liver lesions.

\section{Materials and methods}

Clinical data. A total of 91 patients with liver space-occupying lesions (145 lesions), were inspected by MRI (Signa 3T HDx; GE Healthcare, Chicago, IL, USA) at Zhongnan Hospital of Wuhan University (Wuhan, China) from November 2015 and May 2016. Lesions were collected from all patients, including 
60 males and 31 females, between 26-82 years of age, with an average age of 53 years old. Patients were divided into two groups, according to the presence of benign or malignant lesions. The malignant lesion group (M) consisted of 36 patients (26 males and 10 females), including 22 cases (18 males and 4 females) of liver cancer, and 14 cases ( 8 males and 6 females) of liver metastatic tumor; the benign lesion group (B) consisted of 55 patients (34 males and 21 females), including 36 cases (21 males and 9 females) of liver hemangioma, 11 cases (6 males and 5 females) of liver cyst, 5 cases ( 4 males and 1 female) of focal nodular hyperplasia, and 3 cases (3 males) of liver abscess. The present study was conducted in accordance with the declaration of Helsinki. Ethical approval was gained from the Ethics Committee of Wuhan University (Wuhan, China), and written informed consent was obtained from all study participants.

Scanning method and scan sequences. In order to avoid susceptibility artifacts caused by waste products in organs, each patient was asked to restrict water and dietary intake for $12 \mathrm{~h}$ prior to scanning, and fast on the morning of inspection. Each patient was placed in the supine position in the feet-first-to-head scanning order, and the scanning range covered the entire liver (inferior xiphoid to superior kidney). Transverse-view T1 weighted imaging (T1WI) and T2 weighted imaging (T2WI) routine scanning accompanied by routine fat suppression was performed on all patients. T1WI scanning used the fast spoiled gradient-echo sequence, repetition time (TR), 4,000-4,500 msec; echo time (TE), 2.4-3.8 msec; and field of view (FOV) at $32 \times 37-2 \times 37 \mathrm{~mm}$. T2WI scanning used the fast spin echo-XL sequence, TR, 6,400-6,800 msec; TE, 90.0-92.0 msec; and FOV at 30x30-40x30 mm. infrared-spin-echo echo-planar imaging (SE-EPI) was used for DWI, with b-values at 500, 1,000, and $1,500 \mathrm{sec} / \mathrm{mm}^{2}$, respectively. For three-time scanning (without breath holding); thickness $6 \mathrm{~mm}$, layer spacing $2 \mathrm{~mm}$. The layer varied from 16-20 levels with the b-value changes, and the scanning time was $\sim 230 \mathrm{sec}$.

Image processing. The DWI images were automatically processed by ADW4.4 workstation (GE Healthcare), and ADC values were measured from the solid part of the layer with the greatest diameter. Cystic lesions were measured, avoiding cystic fluid, and focusing on the circular region of interest (ROI), ranging between 100-540 $\mathrm{mm}^{2}$. Particular care was taken to avoid too small or too large ROI, as too small ROI may involve local liquefaction necrosis, and too large ROI may include normal tissue. The measurement was performed three times at different sites, from which the average was calculated.

Statistical analysis. SPSS 13.0 software (SPSS, Inc., Chicago, IL, USA) was used for statistical analysis, and the results were expressed as the mean \pm standard deviation. The average ADC values of benign and malignant space-occupying liver lesions, and the average ADC values of benign and malignant space-occupying liver lesions with normal liver (the ratio of lesion to normal liver, RLN), under the three b-values were compared. The comparison used single-factor analysis of variance, and the Least-Significant Difference test was used for post hoc intergroup analysis to compare differences between groups. The significance level $\alpha$ was 0.05 , and $\mathrm{P}<0.05$ was considered to indicate a statistically significant difference.

\section{Results}

Analysis of patient lesions. Amongst the 91 patients, a total of 145 lesions were identified, including 83 located in the right lobe, 52 located in the left lobe and 10 located in the caudate lobe. The minimum lesion diameter was $\sim 8 \mathrm{~mm}$ and round in shape; the largest lesion diameter was $\sim 46 \mathrm{~mm}$ with a slightly irregular shape. A total of 39 patients presented with multiple lesions, and the remaining patients only presented with a single lesion.

MRI performance. The solid region of primary liver cancer exhibited a slight low- or iso-intensity signal in T1WI whereas the necrotic area exhibited a low signal. In T2WI, 11 cases were associated with partial slight high-intensity signals; 9 with iso-intensity signals and 2 with a circular shadow of low-intensity signals (Fig. 1). The majority of tumors exhibited slight high-intensity signals in DWI. The majority of liver metastatic tumors demonstrated multiple oval low-intensity signals in T1WI, and high-intensity signals in T2WI. Partial high- and low-intensity signals may appear in DWI. The enhanced scanning revealed peripheral enhancement. Liver cysts revealed low intensity signals in T1WI, and significantly high-intensity signals in T2WI (Fig. 2). Hepatic hemangiomas demonstrated low-intensity signals in T1WI, which were higher than in liver cysts, and 'light-bulb signs' high-intensity signals in T2WI (Fig. 3). In DWI, higher-than-liver-parenchyma signals were observed. Focal nodular hyperplasia demonstrated higher-than-liver-parenchyma signals in T1WI, and iso-intensity signals in T2WI, together with partial high-intensity signals (Fig. 4). In DWI, it revealed slight high-intensity signals. The 22 cases of primary hepatocellular exhibited iso-or hypo-signals with hypo-signals in cystic necrotic areas on T1W1, demonstrated hyper-signals (11 cases), iso-signals ( 9 cases), a circular shadow of low-intensity signals ( 2 cases), circular hypo-signals on T2W1 and demonstrated slightly hyper-signals (20 cases), iso-signals (2 cases) or iso-signals on DWI. The 14 cases of liver metastases exhibited multiple circular hypo-signals (12 cases) or slightly circular hyper-signals (2 cases) on T1WI, multiple circular hyper-signals on T2WI and hyper-signals (11 cases) and iso-or hypo-signals on DWI; which all showed annular enhancement in the enhanced scan. The hepatic cysts demonstrated hypo-signals on T1W1, hyper-signals on T2W1, hypo-signals (11 cases) and slightly hyper-signals (1 case) on DWI. Hepatic hemangioma presented hypo-signals on T1W1, which was higher than cyst, and presented hyper-signal on T2W1, called 'Bulb sign'; higher than liver parenchyma of signals on DWI. Hepatic focal nodular hyperplasia (5 cases) presented higher signals than liver parenchyma on T1W1, iso-signals (4 cases) and hyper-signals (1 case) on T2W1, slightly hyper-signals on DWI (Figs. 1-4).

Measurements of ADC values. Statistical analyses of ADC values between liver cancer compared with liver metastatic tumors within the three b-value groups $(500,1,000$ and $1,500 \mathrm{sec} / \mathrm{mm}^{2}$ ) demonstrated statistical significance 


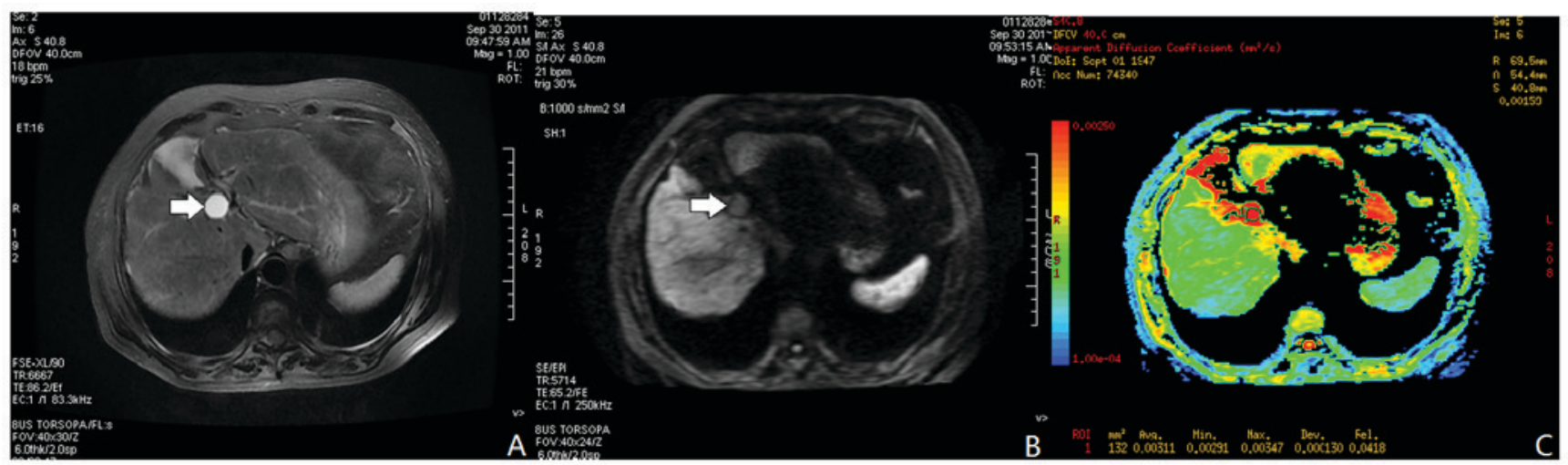

Figure 1. Images from a patient with small cyst. (A) Patient with a small cyst in the right lobe. The fat suppression T2-weighted imaging arrow indicates a circular high intensity signal. (B) The same patient, diffusion weighted imaging $b=1,000 \mathrm{sec} / \mathrm{mm}^{2}$, the arrow indicates the low intensity signal slightly lower than the liver parenchyma. (C) The same patient, apparent diffusion coefficient $=2.51 \times 10^{-3} \mathrm{~mm}^{2} / \mathrm{sec}$.

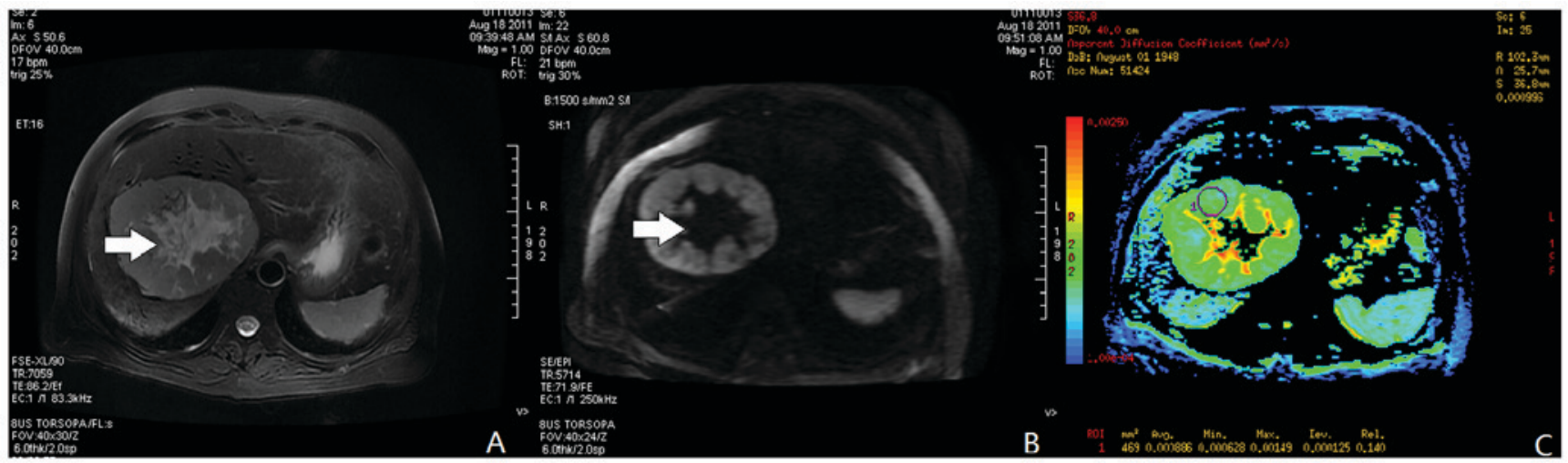

Figure 2. Images of a patient with primary liver cancer. (A) Patient with primary liver cancer in right lobe The fat suppression T2-weighted imaging arrow indicates a bump mixing in signal shadows. (B) The same patient, diffusion weighted imaging $b=1,500 \mathrm{sec} / \mathrm{mm}^{2}$. The arrow indicates mixed signal shadows, the majority of which are higher than the liver parenchyma. (C) The same patient, apparent diffusion coefficient $=1.83 \times 10^{-3} \mathrm{~mm}^{2} / \mathrm{sec}$.

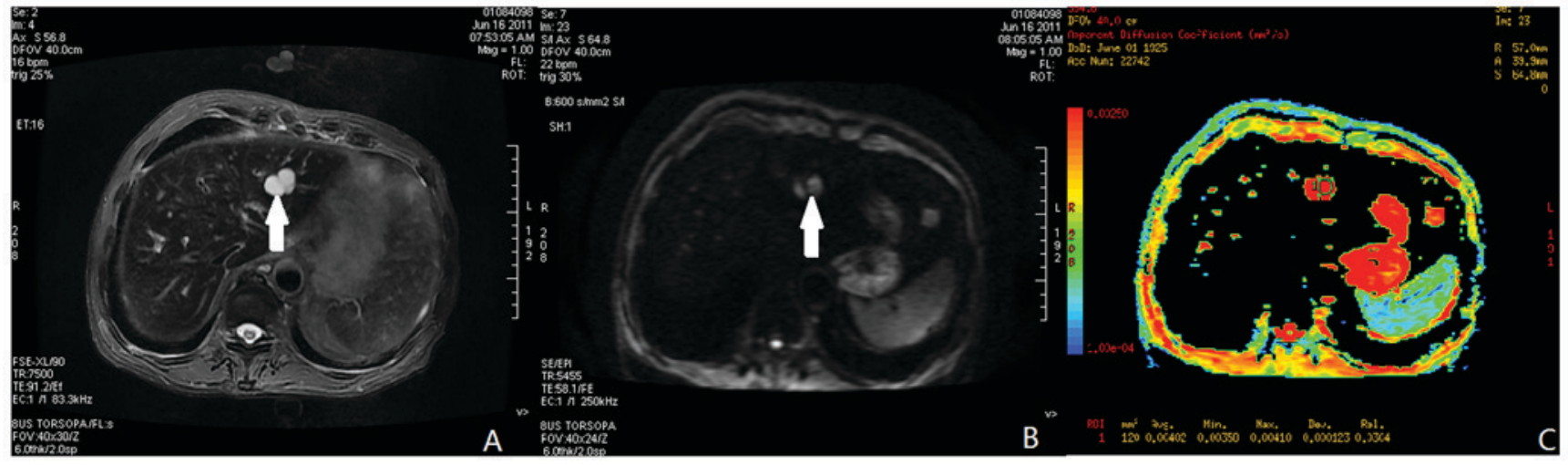

Figure 3. Images of patient with hepatic hemangioma. (A) Patient with hepatic hemangioma in the left lobe. The fat suppression T2-weighted imaging arrow indicates lobulated high intensity signal shadow. (B) The same patient, diffusion weighted imaging $b=500 \mathrm{sec} / \mathrm{mm}^{2}$, the arrow indicates the high intensity signal shadow higher than the liver parenchyma. (C) The same patient, apparent diffusion coefficient $=2.43 \times 10^{-3} \mathrm{~mm}^{2} / \mathrm{sec}$.

differences $(\mathrm{P}<0.05$; Table I). However, in the low b-value group $\left(b=500 \mathrm{sec} / \mathrm{mm}^{2}\right)$, the ADC values were significantly different between malignant lesions, including liver cancer and liver cysts $(\mathrm{P}<0.05)$. ADC values between liver cancer and other malignant lesions, including liver hemangioma, focal nodular hyperplasia (FNH), and hepatic abscesses, revealed no significant differences $(\mathrm{P}>0.05$; Table II), In the middle b-value group $\left(b=1,000 \mathrm{sec} / \mathrm{mm}^{2}\right)$, ADC values were significantly different between liver cancer and liver cysts and between liver hemangioma and liver abscesses $(\mathrm{P}<0.05$; Table II). Furthermore, ADC values between liver metastatic tumors and liver cysts, liver hemangioma, or liver abscesses were also statistically significant $(\mathrm{P}<0.05$; Table II). In the high $b$-value group $\left(b=1,500 \mathrm{sec} / \mathrm{mm}^{2}\right)$, the ADC values were 
Table I. Comparison of mean ADC values between benign and malignant lesions with different b-values in diffusion weighted imaging.

\begin{tabular}{|c|c|c|c|c|c|}
\hline \multirow{2}{*}{$\begin{array}{l}\mathrm{b} \\
\left(\mathrm{sec} / \mathrm{mm}^{2}\right)\end{array}$} & \multicolumn{2}{|c|}{ ADC of malignant lesions $\left(\times 10^{-3} \mathrm{~mm}^{2} / \mathrm{sec}\right)$} & \multicolumn{2}{|c|}{$\mathrm{ADC}$ of benign lesions $\left(\mathrm{x} 10^{-3} \mathrm{~mm}^{2} / \mathrm{sec}\right)$} & \multirow[b]{2}{*}{ P-value } \\
\hline & Primary liver cancer & Liver metastatic tumor & Hepatic hemangioma & Liver cyst & \\
\hline 500 & $2.36 \pm 0.41$ & $2.46 \pm 0.57$ & $2.59 \pm 0.63$ & $2.92 \pm 0.74$ & $<0.05$ \\
\hline 1,000 & $1.99 \pm 0.62$ & $2.17 \pm 0.42$ & $2.23 \pm 0.41$ & $2.51 \pm 0.61$ & $<0.05$ \\
\hline 1,500 & $1.79 \pm 0.67$ & $1.89 \pm 0.78$ & $2.05 \pm 0.55$ & $2.48 \pm 0.41$ & $<0.01$ \\
\hline
\end{tabular}

ADC, apparent diffusion coefficient.

Table II. ADC values of benign and malignant lesions in diffusion weighted imaging with different b-values.

\begin{tabular}{|c|c|c|c|c|c|c|c|}
\hline \multirow[b]{2}{*}{$\mathrm{b}$} & \multicolumn{2}{|c|}{$\mathrm{ADC}$ values of malignant lesions } & \multicolumn{4}{|c|}{$\mathrm{ADC}$ values of benign lesions } & \multirow[b]{2}{*}{ P-value } \\
\hline & $\begin{array}{c}\text { Primary liver } \\
\text { cancer }\end{array}$ & $\begin{array}{l}\text { Liver metastatic } \\
\text { tumor }\end{array}$ & $\begin{array}{c}\text { Hepatic } \\
\text { hemangioma }\end{array}$ & Liver cyst & FNH & $\begin{array}{l}\text { Liver } \\
\text { abscess }\end{array}$ & \\
\hline 500 & $1.45 \pm 0.46$ & $1.47 \pm 0.33$ & $1.56 \pm 0.45$ & $1.86 \pm 0.64$ & $2.48 \pm 0.66$ & $1.52 \pm 0.24$ & $>0.05$ \\
\hline 1,000 & $1.44 \pm 0.45$ & $1.61 \pm 0.49$ & $1.77 \pm 0.36$ & $1.97 \pm 0.58$ & $2.18 \pm 0.52$ & $1.75 \pm 0.36$ & $\mathrm{P}<0.05$ \\
\hline 1,500 & $1.48 \pm 0.46$ & $1.54 \pm 0.39$ & $1.82 \pm 0.61$ & $1.88 \pm 0.52$ & $1.96 \pm 0.56$ & $1.77 \pm 0.61$ & $\mathrm{P}<0.05$ \\
\hline
\end{tabular}

ADC, apparent diffusion coefficient; FNH, focal nodular hyperplasia.

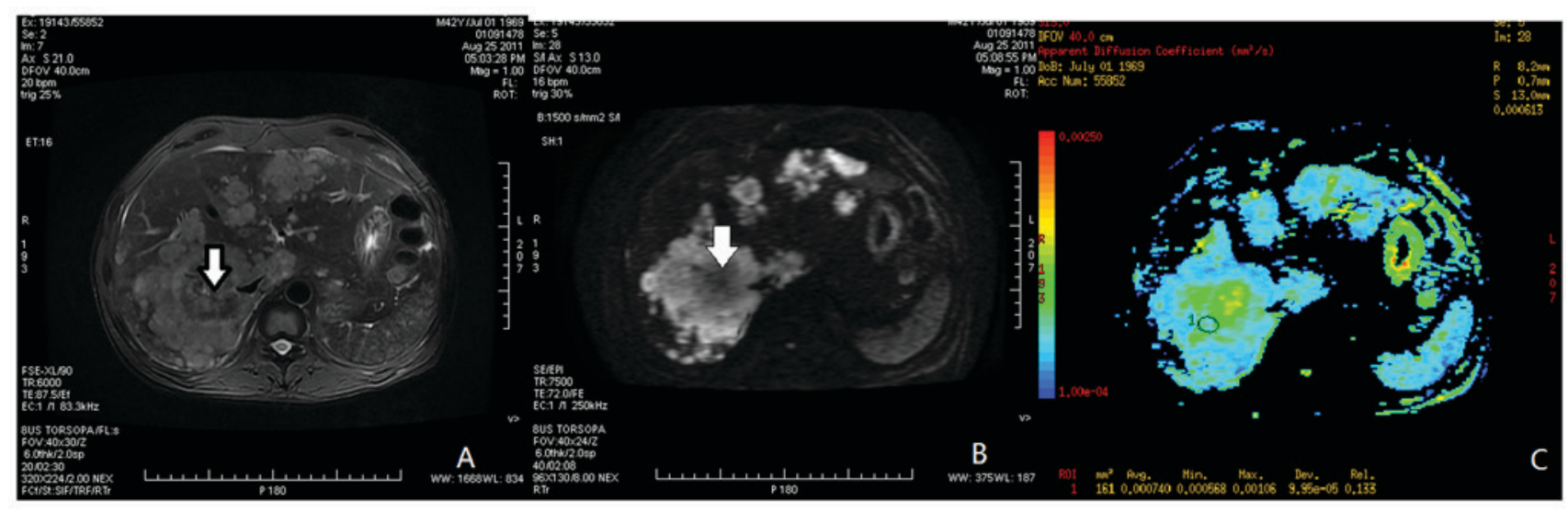

Figure 4. Images of patient with multiple liver metastatic tumors. (A) Patient with multiple liver metastatic tumor. The fat suppression T2-weighted imaging arrow indicates the multiple irregular lumps with a slightly high intensity signal. (B) The same patient, diffusion weighted imaging $b=1,500 \mathrm{sec} / \mathrm{mm}^{2}$. The arrow indicates the high intensity signal shadow higher than the liver parenchyma. (C) The same patient, apparent diffusion coefficient $=1.97 \times 10^{-3} \mathrm{~mm}^{2} / \mathrm{sec}$.

significantly different between liver cancer and liver cysts or liver hemangioma $(\mathrm{P}<0.05)$, and those between liver metastatic tumor and liver cysts or hepatic hemangioma were also statistically significant $(\mathrm{P}<0.05)$. Due to a lack of non-representative samples, statistical analysis of the differences between liver abscesses and FNH was not performed.

Within all three $b$-value groups, including the low b-value group $\left(b=500 \mathrm{sec} / \mathrm{mm}^{2}\right)$, the middle $b$-value group $\left(b=1,000 \mathrm{sec} / \mathrm{mm}^{2}\right)$ and the high $b$-value group $\left(b=1,500 \mathrm{sec} / \mathrm{mm}^{2}\right)$ the ADC values were significantly different between liver cancer compared with benign lesions in all groups at $(\mathrm{P}<0.05, \mathrm{P}<0.05$ and $\mathrm{P}<0.01)$, respectively (Table $\mathrm{I}$ ).
The comparison of ADC values of liver space-occupying lesions with normal liver tissue under different $b$-values is presented in Table II.

The statistical analysis revealed that among the three b-value groups, in the low b-value group ( $\left.b=500 \mathrm{sec} / \mathrm{mm}^{2}\right)$, the $\mathrm{ADC}$ value differences between malignant and benign lesions were statistically significant $(\mathrm{P}<0.05)$; in the middle $b$-value group ( $\left.\mathrm{b}=1,000 \mathrm{sec} / \mathrm{mm}^{2}\right)$, the ADC value differences between malignant and benign lesions were statistically significant $(\mathrm{P}<0.05)$; in the high $\mathrm{b}$-value group $\left(\mathrm{b}=1,500 \mathrm{sec} / \mathrm{mm}^{2}\right)$, the $\mathrm{ADC}$ value differences between malignant and benign lesions were statistically significant $(\mathrm{P}<0.05)$. 


\section{Discussion}

The aim of the present study was to investigate the MRI analysis of malignant and benign liver lesions and normal liver parenchyma. In previous years, with the rapid development and improvement of MRI technologies, and particularly the wide application of SE-EPI detection technology, it is possible to suppress or reduce artifacts caused by physiological motions. Therefore, DWI is able to be effectively used for the diagnosis of liver space-occupying lesions. DWI is a noninvasive method to study the micro-motions of water molecules (17), and may provide further diagnostic information than conventional T1WI and T2WI. This further allows for research on specific aspects of the human body at the micro-level and reflects the spatial organization of human tissue, in addition to the functional status of water molecule exchange among different organs under physiological and pathological conditions. The current application of DWI has been confirmed in the central nervous system $(18,19)$, and the application of DW1 to investigate other parts of the human body. The primary purpose of the present study was to evaluate the application values of DWI and ADC in diagnosing primary liver cancer and liver metastatic tumors.

Different b-values have important impacts on DWI and $\mathrm{ADC}$ values, and the selection of a $\mathrm{b}$-value may determine the size of ADC value (20). In the present study, three different b-values $\left(500,1,000\right.$, and $\left.1,500 \mathrm{sec} / \mathrm{mm}^{2}\right)$ were used for the measurement. Analysis of the results revealed that the lower the $b$-value, the greater the ADC value, and the lower the diffusion weighted signals. In addition, the higher the b-value, the smaller the ADC value, and the higher the diffusion weighted signals, at which point the ADC value is more accurate and closer to the actual $\mathrm{D}$ value. However, a higher b-value requires an increased TE value during scanning, and thus the signal attenuation is more apparent, and at times it may prove difficult to view the images (21). As for the selection of the b-value in DWI towards liver lesions, multiple scholars have obtained different results. A previous study has demonstrated that the b-values within $500-750 \mathrm{sec} / \mathrm{mm}^{2}$ were more appropriate (22). Vandecaveye et al (23) considered a b-value of $500 \mathrm{sec} / \mathrm{mm}^{2}$ to be more appropriate. Another previous study reported that when the $b$-value reached $1,000 \mathrm{sec} / \mathrm{mm}^{2}$ or above, the liver signal attenuation was particularly apparent (24). The results from the present study demonstrated that when the b-value was set at $1,000 \mathrm{sec} / \mathrm{mm}^{2}$, the liver signals demonstrated no significant attenuation. However, when the b-value was set at $1,500 \mathrm{sec} / \mathrm{mm}^{2}$, liver signals in specific cases demonstrated notable attenuation. Notably, in the middle b-value group, in the present study, the differences between liver cysts, liver hemangiomas, liver abscesses, and liver malignant lesions were significant. However, the results in the low- and high b-value groups, with ADC values measured under $b=1,000 \mathrm{sec} / \mathrm{mm}^{2}$ may be more conducive towards the characteristic differentiation of liver space-occupying lesions, consistent with the results of Bruegel et al (25). It is hypothesized that the reasons for the above discrepancies may be caused by different field intensities and settings in different studies, yet the specific reasons and the rational range of the $b$-value still warrants further study.

It was observed throughout the present study that, due to the characteristic differences between benign and malignant liver lesions, the ADC values also differed, with significantly higher ADC values detected for benign lesions compared with malignant lesions. Thus, its mechanisms may be associated with the pathological phenotype and biochemical component changes which occur in these lesions (26). Statistical analysis revealed that the different ADC values observed between benign liver lesions including liver cysts, liver hemangiomas, and liver abscesses (in the middle b-value group; excluding FNH), and malignant lesions including liver cancer, were statistically significant. As DWI uses the diffusion of water molecules inside lesions to diagnose diseases, and due to the tissue component differences in various diseases, the free water content may also be different. The majority of lesions of primary liver cancer and liver metastatic tumors are solid lesions (partial lesions appear as central necrosis, and the ROI points selected in the present study all avoided the cystic necrosis area), lesions may have less free water, making diffusion slow, resulting in a decreased ADC value. Hepatic hemangioma is mainly composed of blood sinusoids and fibrous interval, and because blood sinusoids are full of blood, the physiological movement of water molecules is relatively free and water is able to diffuse rapidly. Therefore, the ADC values obtained are significantly increased compared with those obtained in hepatocellular carcinoma (27). Thus, DWI has a significant value in determining benign and malignant liver space-occupying lesions $(28,29)$, and may further be used to clarify, differentiate, and diagnose malignant and benign liver lesions. In addition, it also provides a noninvasive and reliable clinical adjuvant diagnosis method.

The comparison of ADC values between benign and malignant liver lesions with normal liver parenchyma is named ratio of lesion to normal liver (RLN). Due to patients' individual differences, disease status, size and location of ROI, and different operators, patients may exhibit a wide variation in ADC values even if the $b$-value is the same. The use of RLN may reduce the individual differences between patients' characteristics, including height and weight, but no data demonstrating that RLN is able to distinguish liver malignant tumors and benign lesions has been identified in previous years. In the present study, RLN $<1.6$ was set as the threshold, and the results indicated that the sensitivity, specificity, and accuracy calculated under high $b$-values were higher than those calculated under low b-values. When compared with the settings using ADC $<1.65 \times 10^{-3} \mathrm{~mm}^{2} / \mathrm{sec}$ as the threshold, the specificities obtained under high $b$-values were higher than the latter, but the sensitivities and accuracies were decreased. Therefore, the combination of the two may contribute to improving the identification ability of ADC values towards benign and malignant liver lesions. The results of the present study demonstrated that the differences in RLN between malignant and benign liver lesions were statistically significant, demonstrating more value in differentially diagnosing the features of liver space-occupying lesions than when simply using the ADC values.

Although MRI is expensive and not the preferred method of diagnosis at present, it has a number of advantages as an effective means for identifying suspicious liver space-occupying lesions. MRI is a noninvasive method adopting high soft tissue resolution, multi-dimensional imaging, and large scanning field, which also identifies adjacent organ invasion and large-range lymph node metastasis. The development of MRI, together with advances in technology in the computer field, the 
constant improvement of processing skills, the gradual maturation of coil theories, and the innovations of imaging sequence and post-processing methods has demonstrated considerable progress. Such progress is connected with the research directions of actual clinical needs, and the potential for MRI to be promoted in clinics, thus benefiting more patients with cancer.

To conclude, it was observed that liver space-occupying lesions demonstrate different DWI features and ADC ranges, and DWI accurately determines the nature of lesions, thus exhibiting significance towards the identification of liver benign and malignant space-occupying lesions.

\section{Acknowledgements}

The present study was funded by the National Natural Science Foundation of China (grant nos. 81171315, 81227902).

\section{References}

1. Shibata $\mathrm{T}$ and Aburatani H: Exploration of liver cancer genomes. Nat Rev Gastroenterol Hepatol 11: 340-349, 2014.

2. Parikh T, Drew SJ, Lee VS, Wong S, Hecht EM, Babb JS and Taouli B: Focal liver lesion detection and characterization with diffusion-weighted MR imaging: Comparison with standard breath-hold T2-weighted imaging. Radiolgy 246: 812-822, 2008

3. Ma J Evidence-based guideline: The role of diffusion and perfusion MRI for the diagnosis of acute ischemic stroke: explanation and viewpoint. Chin J Magn Resonan Imag 1: 324-329, 2010.

4. Huang BC, Zhan SH, Geng DY, et al: Diffusion weighted imaging features after hepatic right portal vein ligation in rats. Chin J Magn Reson Imaging: 55-59, 2010.

5. Naminoto T, Yamashita Y, Sumi S, Tang Y and Takahashi M: Focal liver masses: Characterization with diffusion-weighted echoplanar MR imaging. Radiology 204: 739-744, 1997.

6. Koh DM, Collins DJ and Orton MR: Intravoxel incoherent motion in body diffusion-weighted MRI: Reality and challenges. AJR Am J Roentgenol 196: 1351-1361, 2011.

7. Lewis S, Dyvorne H, Cui Y and Taouli B: Diffusion-weighted imaging of the liver: Techniques and applications. Magn Reson Imaging Clin N Am 22: 373-395, 2014.

8. Hu G, Chan Q, Quan X, Zhang X, Li Y, Zhong X and Lin X: Intravoxel incoherent motion MRI evaluation for the staging of liver fibrosis in a rat model. J Magn Reson Imaging 42: 331-339, 2015.

9. Chung SR, Lee SS, Kim N, Yu ES, Kim E, Kühn B and Kim IS: Intravoxel incoherent motion MRI for liver fibrosis assessment: A pilot study. Acta Radiol 56: 1428-1436, 2015.

10. Joo I,Lee JM, Grimm R, Han JK and Choi BI: Monitoring vascular disrupting therapy in a rabbit liver tumor model: Relationship between tumor perfusion parameters at IVIM diffusion-weighted MR imaging and those at dynamic contrast-enhanced MR imaging. Radiology 278: 104-113, 2016.

11. Park YS, Lee CH, Kim JH, Kim IS, Kiefer B, Seo TS, Kim KA and Park CM: Using intravoxel incoherent motion (IVIM) MR imaging to predict lipiodol uptake in patients with hepatocellular carcinoma following transcatheter arterial chemoembolization: A preliminary result. Magn Reson Imaging 32: 638-646, 2014.

12. Wang M, Li X, Zou J, Chen X, Chen S and Xiang W: Evaluation of hepatic tumors using intravoxel incoherent motion diffusion-weighted MRI. Med Sci Monit 22: 702-709, 2016.

13. Doblas S, Wagner M, Leitao HS, Daire JL, Sinkus R, Vilgrain V and Van Beers BE: Determination of malignancy and characterization of hepatic tumor type with diffusion-weighted magnetic resonance imaging: Comparison of apparent diffusion coefficient and intravoxel incoherent motion-derived measurements. Invest Radiol 48: 722-728, 2013.

14. Watanabe H, Kanematsu M, Goshima S, Kajita K, Kawada H, Noda Y, Tatahashi Y, Kawai N, Kondo H and Moriyama N: Characterizing focal hepatic lesions by free-breathing intravoxel incoherent motion MRI at 3.0 T. Acta Radiol 55: 1166-1173, 2014.
15. Colagrande S, Regini F, Pasquinelli F, Mazzoni LN, Mungai F, Filippone A and Grazioli L: Focal liver lesion classification and characterization in noncirrhotic liver: a prospective comparison of diffusion-weighted magnetic resonance-related parameters. J Comput Assist Tomogr 37: 560-567, 2013.

16. Zhu L, Cheng Q, Luo W, Bao L and Guo G: A comparative study of apparent diffusion coefficient and intravoxel incoherent motion-derived parameters for the characterization of common solid hepatic tumors. Acta Radiol 56: 1411-1418, 2015.

17. Kandpal H, Sharma R, Madhusudhan KS and Kapoor KS: Respiratory-triggered versus breath-hold diffusion-weighted MRI of liver lesions: Comparison of image quality and apparent diffusion coefficient values. AJR Am J Roentgenol 192: 915-922, 2009.

18. Jungmann PM, Baum T, Schaeffeler C, Sauerschnig M, Brucker PU, Mann A, Ganter C, Bieri O, Rummeny EJ, Woertler K and Bauer JS: 3.0T MR imaging of the ankle: Axial traction for morphological cartilage evaluation, quantitative T2 mapping and cartilage diffusion imaging-A preliminary study. Eur J Radiol 84: 1546-1554, 2015.

19. Tang Y, Wang H, Wang Y, Li J, Jia R, Ma L and Ye H: Quantitative comparison of MR diffusion-weighted imaging for liver focal lesions between 3.0T and 1.5T: Regions of interest of the minimum-spot ADC, the largest possible solid part and the maximum diameter in lesions. J Magn Reson Imaging 44: 1320-1329, 2016.

20. Gourtsoyianni S, Papanikolaou N, Yarmenitis S, Maris T, Karantanas A and Gourtsoyiannis N: Respiratory gated diffusion-weighted imaging of the liver: Value of apparent diffusion coefficient measurements in the differentiation between most commonly encountered benign and malignant focal liver lesions. Eur Radiol 18: 486-492, 2008.

21. Kakite S, Hadrien D, Besa C, Cooper N, Facciuto M, Donnerhack C and Taouli B: Hepatocellular carcinoma: Short-term reproducibility of apparent diffusion coefficient and intravoxel incoherent motion parameters at 3.0T. J Magn Reson Imaging 41: 149-156, 2015.

22. Yoshikawa T, Kawamitsu H, Mitchell DG, Ohno Y, Ku Y, Seo Y, Fujii $\mathrm{M}$ and Sugimura K: ADC measurement of abdominal organs and lesions using parallel imaging technique. AJR Am J Roentgenol 187: 1521-1530, 2006.

23. Vandecaveye V, De Keyzer F, Verslype C, Op de Beeck K, Komuta M, Topal B, Roebben I, Bielen D, Roskams T, Nevens F and Dymarkowski S: Diffusion-weighted MRI provides additional value to conventional dynamic contrast-enhanced MRI for detection of hepatocellular carcinoma. Eur Radiol 19: 2456-2466, 2009.

24. Taouli B, Vilgrain V, Dumont E, Daire JL, Fan B and Menu Y: Evaluation of liver diffusion isotropy and characterization of focal hepatic lesions with two single-shot echo-planar MR imaging sequences: Prospective study in 66 patients. Radiology 226: 71-78, 2003.

25. Bruegel M, Holzapfel K, Gaa J, Woertler K, Waldt S, Kiefer B, Stemmer A, Ganter C and Rummeny EJ: Characterization of focal liver lesions by ADC measurements using a respiratory triggered diffusion-weighted single-shotecho-planarMRimaging technique. Eur Radiol 18: 477-485, 2008.

26. Ma C, Liu L, Li J, Wang L, Chen LG, Zhang Y, Chen SY and Lu JP: Apparent diffusion coefficient (ADC) measurements in pancreatic adenocarcinoma: A preliminary study of the effect of region of interest on ADC values and interobserver variability. J Magn Reson Imaging 43: 407-413, 2016.

27. Bruegel M, Muenzel D, Waldt S, Specht K and Rummeny EJ: Hepatic epithelioid hemangioendothelioma: Findings at CT and MRI including preliminary observations at diffusion-weighted echo-planar imaging. Abdom Imaging 36: 415-424, 2011.

28. Ertas G, Onaygil C, Akin Y, Kaya H and Aribal E: Quantitative differentiation of breast lesions at 3T diffusion-weighted imaging (DWI) using the ratio of distributed diffusion coefficient (DDC). J Magn Reson Imaging 44: 1633-1641, 2016.

29. Erturk SM, Ichikawa T, Sano K, Motosugi U, Sou H and Araki T: Diffusion-weighted magnetic resonance imaging for characterization of focal liver masses: Impact of parallel imaging (SENSE) and b value. J Comput Assist Tomogr 32: 865-871, 2008.

This work is licensed under a Creative Commons Attribution-NonCommercial-NoDerivatives 4.0 International (CC BY-NC-ND 4.0) License. 